Journal of Applied Fluid Mechanics, Vol. 9, Special Issue 1, pp. 69-80, 2016.

Selected papers from the $7^{\text {th }}$ International Exergy, Energy

and Environment Symposium, IEEE7-2015

Available online at www.jafmonline.net, ISSN 1735-3572, EISSN 1735-3645.

DOI: $10.36884 / \mathrm{jafm} .9$. SI1.26397

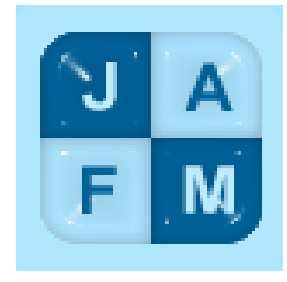

\title{
Scavenging Process Analysis in a 2-Stroke Engine by CFD Approach for a Parametric 0D Model Development
}

\author{
S. Cagin ${ }^{1,2}$, N. Bourabaa ${ }^{3}$, E. Delacourt, ${ }^{3,4}$, C. Morin ${ }^{3,4}$, X. Fischer ${ }^{1,2}$, D. Coutellier ${ }^{3,4}$, B. \\ Carré $^{5}$ and S. Loumé \\ ${ }^{1}$ Université de Bordeaux, I2M CNRS UMR 529533607 Bordeaux, France \\ ${ }^{2}$ ESTIA Recherche 64210 Bidart, France \\ ${ }^{3}$ UVHC, ENSIAME Le Mont Houy 59313 Valenciennes cedex 9, France \\ ${ }^{4}$ UVHC, LAMIH CNRS UMR 8201 Le Mont Houy 59313 Valenciennes cedex 9, France \\ ${ }^{5}$ AKIRA Technologies, ZA Saint Frédéric rue de la Galupe 64100 Bayonne, France \\ †Corresponding Author Email: celine.morin@univ-valenciennes.fr
}

(Received October 20, 2015; accepted December 10, 2015)

\begin{abstract}
This paper presents a method to improve cylinder design of 2-stroke auto-ignition engine based on a CFD (Computational Fluid Dynamics) study of internal flows in the chamber and an unsteady global 0D parametric approach. In 2-stroke engine, scavenging process plays an important role regarding engine efficiency and pollutant emissions. Several geometrical and environmental parameters (like piston velocity and inlet/outlet thermofluid conditions) impact the scavenging process and most of them vary when the engine is running. To improve the scavenging process, an analytical model (integrating design parameter variations) is developed and will be implemented in OD global model. CFD simulations are used to establish the analytical scavenging model. The CFD model includes species transportation, piston motion (remeshing), turbulent effectsbut it does not take into account the combustion process or the aerodynamics in the cylinder before the beginning of scavenging. After defining the influent parameters on the scavenging, multiple simulations with varying values of parameters were run and a data base was created. The data base will be used to develop a reduced model of the scavenging process which will be integrated in a global 0D model of the engine. Through a reference case, the in-cylinder flow is analyzed and the evolution of velocity, pressure, species and turbulent kinetic energy fields during scavenging are discussed. After a statistical treatment, the results of simulations highlight two main significant parameters: the advance of intake opening and the angle of the intake duct. The decoupling of these two parameters is particularly suitable for the optimization of engines.
\end{abstract}

Keywords: 2-stroke engine; Scavenging; CFD model;Compressible flow;Species transportation;Statistical treatment.

\section{NOMENCLATURE}

Subscripts
adv advance
ch charging
exh
exhaust

\section{INTRODUCTION}

Due to emissions standards more and more drastic and fuel consumption constraints, Internal Combustion Engines (ICE) used for transportation arewidely studied to improve efficiency and obtain better performances. Despite decades of development, their mean efficiency is still low and

$\begin{array}{ll}\text { in } & \text { intake } \\ \mathrm{sc} & \text { scavenging } \\ \mathrm{tr} & \text { trapping }\end{array}$

improvements can be made in terms of design and performances.

Two-stroke compression ignition engines and the enhancement of the cylinder aerodynamics are particularly of great interests. The performances of engine depend on a lot of factors such as the quantity of injected fuelorpower duration. One of crucial issues is the aerodynamics in the combustion chamber controlling the quality of scavenging and 
the composition of gases available for combustion process. Indeed, scavenging is the process by which the fresh gases come into the combustion chamber, pushing through the exhaust ports the burnt gases. It plays an important role in the performance, the pollutant emissions and engine efficiency.

Despite its low efficiency, automotive industry has recently a renewed interest for 2-stroke engines thanks to its advantages. Smaller and lighter than 4-strokeengines, 2-strokes engines fireonce every revolution offering higher power, greater and smoother torque (Mattarelli, 2009; Trescher, 2008). The interest is also linked to the development of new technologies approaches which increase their efficiencies. Monitoring and evaluation of performances in real time have revolutionized the engine design. But, to define the best design and to manufacture these engines, it is essential to study the engine behavior and to test some configurations.

Two-stroke engines have suffered from high emission and poor fuel economy compared to more efficient 4-stroke engines. This is particularly true for 2-strokeengineswith ports where there is no possible variation of the opening angles (intake and exhaust crank angles degrees (CAD) less adaptable). Moreover, the exhaust port always opens first in order to avoid any backflow (Mattarelli, 2009)and therefore closes last. This way, the burnt gases at high pressure in the chamber flow through the exhaust ports and the cylinder pressure decreases. When the intake port opens, fresh gases enter (if the pressure difference is favorable). But, the consequence is also that after closing the intake ports, the exhaust one is still opened and gases still flow through it.

In addition of possible backflow, the main difficulties with the 2-stroke engine and a scavenging process by ports are the mixture of burnt and unburnt gases and the short-cutting of the fresh charge as Lamas Galdo and Rodriguez Vidal (2012a) underline.Compared to the others, the short-cuttingisthe most problematic issue. It characterizes the inlet and exit of fresh gases before the end of the scavenging process, which reduces the engine efficiency. Short-cutting impact is particularly undesirable for spark-ignition engines because gasoline is incorporated into fresh gases. That is why its modelling is widely studied (Honget al., 2004). One of the first solutions used to reduce the short-cutting was the addition of deflectors on the piston head. It offered the advantage to significantly improve the scavenging efficiency without any important design change. But, the evolution of piston head and especially the development of piston bowls (for direct injection systems) reducing the pollutant emission (Prasadet al., 2011) made this solution obsolete. Other possibilities stay available such as the orientation of ducts and ports.

0D models are developed to improve the understanding of engine behavior and to facilitate their design. They are systemic models based on simplified physics laws. All variables are only time-dependent. The studied system is divided into several subsystems (e.g. combustion chamber, moving parts, cylinder head, turbocharger/supercharger, pneumatic lines, etc). Each subsystem is considered as a point where all variables (pressure, temperature, etc) are solved. All subsystems are linked together influencing the behavior of surrounding components. These simplifications allow the simulations to run very fast, by including interdependencies between subsystems but with less accuracy than a CFD approach. Many models are developed in order to improve 0D accuracy.Agoodmodelling of scavenging process in $0 \mathrm{D}$ is essential to know the composition of gases in the cylinder especially for the combustion process. Scavenging modellingimplies the localization of the gases in the cylinder and the composition of entering and leaving gases,which is complex when no spatial dimension is taken into account. That is why some authors couple models of different dimensionstogether in order to take the advantages of fast low dimension models and of accurate high dimension ones as done by Mattarelli (2009). He coupled 1D and multi-dimensional CFD simulations. The three models always communicate during the simulations, providing the inputs of other models. Compromises between duration and accuracy need to be done.

Many CFD studies of engines exist. All intake and exhaust configurations are studied:Kato et al. (1991),Honget al.(2004), Pittaet al. (2008), Nooret al. (2008)andMattarelli (2009) were foccused on flow phenomena in engines with ports; Trescher (2008)and Ingvorsenet al.(2012) were interesting by engines with ports (intake) and valves (exhaust) configuration. They mostly concerned small 2-stroke and 4-stroke engines because of automotive industry needs but they are also used for large marine engines(Lamas Galdo and Rodriguez Vidal, 2012a). Despite technologies and computers evolutions, CFD calculations stay expensive in resources and time. That explains why most of studies are focused on one particular phenomenon, commonly combustion process and pollutant emissions.Indeed, the antipollution norms are more and more restrictive and drastic which encourages to perfectly understand and to control the combustion.

However, even if the combustion process is the most influent process in pollution, the scavenging hasadeterminant role in pollutants formation and also in engine performances and efficiency. Scavenging impacts both the composition of gases before the beginning of the combustionand the aerodynamics of the gases in the cylinder which are two important parameters in pollution control. But only few papers are focused on the CFD of the scavenging process like Mattarelli (2009), especially in engines with ports whereas it is very problematic.Mattarelli(2009) discusses scavenging results depending on the transfer port elevation (the angle of the port axis as regards the horizontal plane) and then compares the 2-stroke engine configuration with 4-stroke one for different loads.

In this work, a systemic approach and 0D models 
appear more adapted to test multiple designs or to achieve a preliminary design phase. They also have the advantage to take into account all the components of engine environment and their complex interactions. But $0 \mathrm{D}$ models have no spatial dimension: a new model of the scavenging process needs to be integrated in this modelling. Both OD and CFD modellings are used but for different goals. As a scavenging model is needed in the 0D modelling, CFD models are implemented in order to provide information about scavenging process. Multiple cases with varying parameters (angles, pressure...) are used to quantify the scavenging efficiency and the composition of gases in the chamber according to geometrical and environmental parameters. Thanks to CFD results, a general model of scavenging will be developed and integrated in the $0 \mathrm{D}$ model. In order to save time testing the method with simple cases, first CFD models were in $2 \mathrm{D}$, to validate the method and provide first results about the scavenging process.

The numerical model with the governing equations involved in the numerical model is introduced. Then, the characteristics of in-cylinder flow and its evolution during the scavenging process are presented in terms of pressure, velocity, species and turbulent kinetic energy. Finally, after a statistical treatment of scavenging efficiency results, the most influential parameters in 2D are highlighted.

\section{THE REFERENCE CASE}

The present study is focused on the scavenging process. The complexity of combustion leaded to not include it in the model. The scavenging process is assumed to occur sufficiently long time after the beginning of the combustion; and the combustion is considered as total.To set up the initial pressure and temperature conditions in the cylinder, a Matlab ${ }^{\circledR}$ program is used. A classical and simplified combustion law, the heat release Wiebe law, expressed by Ghojel(1997), is coded in the program.

Asall the cylindersare identical, only one cylinder has been taken intoaccountintheCFDapproach, the aim being toreduce the computational effort. The possible effects of the acoustic waves and the disturbances cylinder to cylinder will not be covered here.

\subsection{Governing equations}

Computational Fluid Dynamics is the analysis of systems involving fluid flow, heat transfer such as chemical reactions by means of computer-based simulations. This technique is very powerful and currently widely used in industrial and non-industrial applications. This numerical technique is based on the resolution of transport equations written in a conservative form. Because of significant commonalities between the various equations, they can be represented by a transport equation containing the unsteady, the convective, the diffusive and the source terms:

$\frac{\partial}{\partial t}(\rho \phi)+\operatorname{div}(\rho \vec{V} \phi)=\operatorname{div}\left(\Gamma_{\phi} \operatorname{grad} \phi\right)+S_{\phi}$ where $\phi$ is a general variable of the flow including velocity components, temperature, species concentration, $\Gamma_{\phi}$ is diffusion coefficient and $S_{\phi}$ all the sources of $\phi$. Equation (1) is used as the starting point for computational procedures in the finite volume method.

Flows in laminar regime are completely described by Eq. (1) written for continuity and Navier-Stokes equations, energy, but flows are mostly turbulent in practical cases.

The turbulence is an uncertain, chaotic, unsteady and unpredictable phenomenon. In engines, the air flow is fully turbulent and is characterized by large scales of length and energy (Lamas Galdo and Rodriguez Vidal, 2012). The transport equations are then solved in unsteady cases. The variables of the flow are decomposed according the Reynolds decomposition into a steady mean value Фand a fluctuatingcomponent $\phi^{\prime}(\mathrm{t}): \phi(\mathrm{t})=\Phi+\phi^{\prime}(\mathrm{t})$.

In order to capture all the scales, the more accurate solution is the direct approach called DNS (Direct Numerical Simulation) which is costly in time and resources.

consistsofsolvingthetransportequationsinunsteadyre gimeusingveryfinemeshes. The industrial constraints of development make this approach unusable in most cases.

Other alternative methods can be used to solve the Navier-Stokes equations in case of turbulent regimes. The first one is based on spatial or temporal filtrations of the turbulent structures: every structure with a scale more important than the filter is computed whereas the structures under the filter are modeled. Nevertheless, this approach called LES (Large Eddy Simulation) is also time consuming because the simulation is carried out in unsteady regime with very fine meshes.

The most used approach in engineering applications is the RANS one (Reynolds-averaged Navier-Stokes) which offers the best compromise between time calculation and accuracy. This approach applies the Reynolds decomposition first and then an average operation of the transport equations. Both filtering and averaging methods introduce additional unknown terms in these averaged equations presented here below:

- The continuity equation:

$\frac{\partial \rho}{\partial t}+\frac{\partial}{\partial x_{i}}\left(\rho U_{i}\right)=0$

- The momentum equation:

$\frac{\partial}{\partial t}\left(\rho U_{i}\right)+\frac{\partial}{\partial x_{j}}\left(\rho U_{i} U_{j}\right)=-\frac{\partial P}{\partial x_{i}}+\frac{\partial \tau_{i j}}{\partial x_{j}}+\frac{\partial}{\partial x_{j}}\left(-\rho \overline{u_{\imath}^{\prime} u_{j}^{\prime}}\right)$

As the fluid is considered as Newtonian, the components of the Reynolds stress tensor are expressed as: 
$\tau_{i j}=\mu\left(\frac{\partial U_{i}}{\partial x_{j}}+\frac{\partial U_{j}}{\partial x_{i}}-\frac{2}{3} \delta_{i j} \frac{\partial U_{k}}{\partial x_{k}}\right)$

- The energy equation:

$$
\begin{gathered}
\frac{\partial}{\partial t}(\rho E)+\frac{\partial}{\partial x_{j}}\left[U_{i}(\rho E+P)\right]= \\
\frac{\partial}{\partial x_{j}}\left[\left(k_{t}+\frac{C_{p} \mu_{t}}{P r}\right) \frac{\partial T}{\partial x_{j}}+u_{i} \tau_{i j}\right]
\end{gathered}
$$

The additional terms $-\rho \overline{u_{\imath}^{\prime} u_{\jmath}^{\prime}}$ called Reynolds stress represents the turbulence effects and needs to be modeled in order to achieve the closure' of the model. The number of added equations is variable (1 to 7 ) and allows to classify the models in different categories depending on the number of these additional equations.

- The stateequation:PressurePand temperature $\mathrm{T}$ are high in the cylinder; both densities pof burnt and unburnt gases follow the ideal gas law:

$\rho=\frac{P}{r T}(6)$

with $\mathrm{r}$ the specific gas constant.

\subsection{Turbulence modelling}

As seen in the previous section, the average operation of the transport equations transforms the initial closed problem into an opened one containing more unknown variables than equations. The first added term is the Reynolds stress in Eq.(2). To compute it,the common method is based on the Boussinesq hypothesis which allows to relatethe Reynolds stress to the mean velocity gradients:

$$
-\rho u_{i}^{\prime}=\mu_{t}\left(\frac{\partial U_{i}}{\partial x_{j}}+\frac{\partial U_{j}}{\partial x_{i}}\right)-\frac{2}{3}\left(\rho k+\mu_{t} \frac{\partial U_{k}}{\partial x_{k}}\right) \delta_{i j}
$$

The advantage of this hypothesis is the relatively low computational cost associated with the computation of the turbulent viscosity $\mu_{\mathrm{t}}$. But, in addition of the turbulent viscosity $\mu_{\mathrm{t}}$, Eq. (7) also introduces two new terms: the turbulent kinetic energy $\mathrm{k}$ and its dissipation rate $\varepsilon$. These terms allow a full characterization of the turbulent flow.The RANS turbulent models add the extra transport equations needed to close the problem and to characterize the turbulent phenomena in the flow, depending on the RANS model selected, from 1 to 5 transport equations in 2D (from 1 to 7 equations in 3D).

The most used models are the "two equations" ones thanks to a good compromise between time calculations andresults accuracies.

Regarding the case of study, the $\mathrm{k}-\varepsilon$ model offers the advantage of a better modelling for flows involving rotation, boundary layers under strong adverse pressure gradients, separation, and recirculation.
The two transport equations respectively related to the turbulent kinetic energy $\mathrm{k}$ and the turbulence dissipation rate $\varepsilon$ are:

$$
\begin{gathered}
\frac{\partial}{\partial t}(\rho k)+\frac{\partial}{\partial x_{i}}\left(\rho k U_{i}\right)=\frac{\partial}{\partial x_{j}}\left[\alpha_{k} \mu_{t} \frac{\partial k}{\partial x_{j}}\right] \\
+G_{k}+G_{b}-\rho \varepsilon-Y_{M} \\
\frac{\partial}{\partial t}(\rho \varepsilon)+\frac{\partial}{\partial x_{i}}\left(\rho \varepsilon U_{i}\right)=\frac{\partial}{\partial x_{j}}\left[\alpha_{\varepsilon} \mu_{t} \frac{\partial \varepsilon}{\partial x_{j}}\right] \\
+C_{1 \varepsilon} \frac{\varepsilon}{k}\left(G_{k}+G_{3 \varepsilon} G_{b}\right)-\rho C_{2 \varepsilon} \frac{\varepsilon^{2}}{k}
\end{gathered}
$$

In Eq. (8) and (9), $G_{k}$ and $G_{b}$ represent the generation of turbulent kinetic energy due to the mean velocity gradients and to the buoyancy respectively. $\mathrm{Y}_{\mathrm{M}}$ represents the contribution of the fluctuating dilatation in compressible turbulence to the overall dissipation rate. $\mathrm{C}_{1 \varepsilon}, \mathrm{C}_{2 \varepsilon}, \mathrm{C}_{3 \varepsilon}, \alpha_{\varepsilon}$ and $\alpha_{\mathrm{k}}$ are the constants of the model; they were experimentally set to 1.44 for $\mathrm{C}_{1 \varepsilon}, 1.9$ for $\mathrm{C}_{2 \varepsilon}, 1$ for $\alpha_{\varepsilon}$ and 1.2 for $\alpha_{k}$. These values are suitable for most of flows. But they can be changed to improve the numerical results.

The turbulent viscosity is not an inherent property of the fluid itself but of the turbulent flow. It is directly linked to the scales of motions and energy. So, the turbulent viscosity relies $\mathrm{k}$ and $\varepsilon$ as follows:

$\mu_{t}=\rho C_{\mu} \frac{k^{2}}{\varepsilon}$

A comparison of several turbulence models have been done by Sigurdsson (2011) and Zancanaro and Vielmo (2010) and both studies conclude that there are no significant differences in the results of the scavenging process by varying the turbulence model. The k- $\varepsilon$ Realizable model (Shihet al., 1994) has been chosen for this study. The term "realizable" means that the model satisfies certain mathematical constraints on the Reynolds stress, consistent with the physics of turbulent flows which motivated its use. This is not the case of the standard k- $\varepsilon$ model neitherthe RNG k- $\varepsilon$ model.

For the near-wall turbulence treatment, the enhanced wall option has been used. It combines a two-layer model with empirical wall functions depending on the mesh size and the wall shearing stress.

\subsection{Fresh and burnt gases modelling}

The objective of this study is the optimization of the scavenging process in a combustion chamber using a CFD approach. As presented above, the combustion was not modeled in order to decrease calculation time. Nevertheless, the scavenging characterization requires the knowledge of flow structures for fresh and burnt gases as well as their parameters (pressure, velocities).

Another alternative consists in modelling the fresh gases by a cold flow and the burnt gases by a hot one. In opposition of two-phase flows modelling, 
this approach reduces the calculations time. But, a flow of single fluid having different temperature levels leads to obtain a gas mixture and the results do not allow to quantify the various parameters (such as the mass fraction of burnt and fresh gases) necessary for the scavenging optimization.

A simplified approach was therefore used in this study. It consists in representing the fresh gas by asinglespeciesand the burnt gas by another. Theaveragecomposition of fresh and burnt gases has been taken into account in order to provide the average values of each species features (density, viscosity...).In CFD, this hypothesis leads to solve additional scalar equations for the transport of chemical species based on the molar or mass fraction of the species as written here below:

$\frac{\partial}{\partial \mathrm{t}}\left(\rho \mathrm{C}_{\mathrm{i}}\right)+\frac{\partial}{\partial \mathrm{x}_{\mathrm{j}}}\left(\mathrm{U}_{j} \rho \mathrm{C}_{\mathrm{i}}\right)=-\frac{\partial}{\partial \mathrm{x}_{\mathrm{j}}}\left(\mathrm{J}_{i}\right)_{j}$

With $\overrightarrow{J_{l}}$ the mass diffusion flux of speciesi.In the case of two chemical species, only one equation is solved for one speciesallowing to obtain the mass concentration $\mathrm{C}_{1}$. As the sum of the mass concentrations is equal to one, the second value $C_{2}$ is deduced from the balance equation:

$\mathrm{C}_{2}=1-\mathrm{C}_{1}$

This approach has the advantage of solving only one additional equation while the combustion modeling would require the solution of transport equations for all the chemical species present in the products and the reactants.It is then possible to calculate the flow rate of the fresh and burnt gases by evaluating the flow of chemical species in different areas of the computational domain.

\subsection{Numerical mode}

The numerical model is created using the commercial software Ansys ${ }^{\circledR}$ Fluent ${ }^{\circledR} 14.5 .7^{1}$, based on the finite volume method. The numerical results are obtained by solving the governing and turbulence equations exposed in previous sections. The solving algorithm used is the velocity-pressure coupling one, well adapted to compressible and turbulent flows. The discretization scheme for both flows and flow gradients is $2^{\text {nd }}$ order upwind scheme. Radiative effects are not modeled because they mainly occur during the combustion and the soot production, phenomena not studied in this work. Convection and conduction are integrated in energy equation. At least, first order implicit discretization was used for the temporal treatment, with a constant time step.

\subsection{Computational domain of the reference case}

All dimensions (cf. Fig.1) are taken on the basis of the 3D geometry. All the following values are the same whatever the case is: piston diameter $\mathrm{D}=82 \mathrm{~mm}$, conrod length $=180 \mathrm{~mm}$, crank length $=45.4 \mathrm{~mm}$, intake and exhaust ducts lengthL $=100 \mathrm{~mm}$, sloping ducts length $\mathrm{l}=50 \mathrm{~mm}$. The

${ }^{1}$ ANSYS Inc., Canonsburg, Pennsylvania piston displacement is given by a motion law expressed by Swoboda(1984).

Cylinder

D

Piston

$\mathrm{L}$

$\mathrm{H}$

Intake

BDC

Fig. 1. Cylinder geometry.

A very simplified geometry in $2 \mathrm{D}$ has been chosen to get first results, to test and to validate the approach used. Then, this approach will be used with a complex 3D geometry.

The simulation begins just before opening exhaust port and ends just after closing the port. This way, the duration of the simulation is reduced to its minimum. In order to compare all simulations results, the start angle is the same for all cases: 94 CADafter TDC (Top Dead Center). Engine velocity is set to $2500 \mathrm{rpm}$ so $\mathrm{H}$ is equal to $36.06 \mathrm{~mm}$.

The spatial discretization is a crucial step of the modelling. Because of 2D simple geometry and no valve is used, the mesh is only made of hexahedral elements. In addition of their precision and stability, they have the advantage to facilitate the removal/creation of cells in the dynamic mesh process. Indeed, the piston motion involves the creation of new cells until it reaches the BDC (Bottom Dead Center) and the removal of some during piston rise.The numerical algorithm implemented for the dynamic mesh automatically updates the mesh after each time step and associated piston motion using a meshing tool called "layering". This method consists on adding or removing layers of cells adjacent to a moving boundary (piston head in our case) based on the height of the layer adjacent to the moving surface. The procedure is shown inFig. 2.
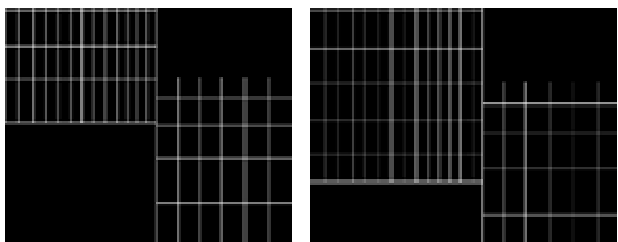

Fig. 2. Remeshing.

The size of mesh elements influences the precision of the numerical model results but also the solving time. In order to find the more suitable size, different meshes were tested. The result, presented by Caginet al. (2014), shows that 10 elements per centimeter provide the best results. But, the near-wall refinementimposes smaller elements close to the walls. During the simulation, the number of elements varies between 20000 cells and 50000 
cells depending on the experiment configuration and piston position.

As explained before, only two types of species are modelled: fresh and burnt gases. The two species are considered homogeneous in terms of pressure, temperature, composition in their respective areas. The combustion reaction is considered as stoichiometric and complete. These hypotheses lead to consider only fresh gases in the intake duct and only burnt gases in the cylinder and the exhaust duct. The same distribution of species has been done by Lamas Galdo and Rodriguez Vidal (2012b) or Nooret al. (2008). With Diesel fuel used in the engine, the proportion of species in the cylinder before the combustion corresponds to the stoichiometric conditions (AFR - Air-Fuel Ratio around 14.6). In Diesel engines, there is an excess of fresh gases in the cylinder most of time. Stoichiometric proportions correspond to a full load of the engine. It is a critical phase of use of the engine which is interesting to study.

The combustion process is not the object of the study so the initial conditions in the cylinder are determined by a Matlab ${ }^{\circledR}$ program and the Wiebe law. The conditions are determined in order to have a maximum pressure of $150 \mathrm{bar}$ in the cylinder for an engine velocity of 2700rpm (expected conditions with full load) and 120bar for an engine velocity of 2300rpm (typical conditions).

The intake and exhaust boundary conditions are set respectively to $333.15 \mathrm{~K}$ and $873.15 \mathrm{~K}$ for all simulations. The pressure is a varying parameter. The pressure and temperature also correspond to the initial conditions in the ducts.

For the initial conditions in the cylinder, the pressure was set to $10.8 \mathrm{bar}$ and the temperature to 1912K (values provided by the Matlab ${ }^{\circledR}$ program).

\section{EXPERIMENTAL STUDY}

In order to validate the numerical model, a comparison between experimental and numerical results has been done.

\subsection{Wind tunnel}

Geometry of combustion chamber has been designed and produced in ABS (Acrylonitrile Butadiene Styrene) thermoplastic (cf. Fig. 3).

This choice of material for the experimental cylinder had two main restrictions on possible trials: no combustion occurs because materials cannot bear the extreme pressure and temperature conditions met in the cylinder; no piston motion is possible because the plastic would melt with friction heatings. All tests will be done for a determined position of piston.

\subsection{Test procedure}

Two different positions of piston have been selected. First, the piston is at BDC.Second, the piston half-covered the intake ports, this position will be referred as "middle point" in the following sections. Thanks to doubled measurement equipment and automatic standardization, the wind tunnel provides an accurate mass flowrate and inlet/outlet pressures
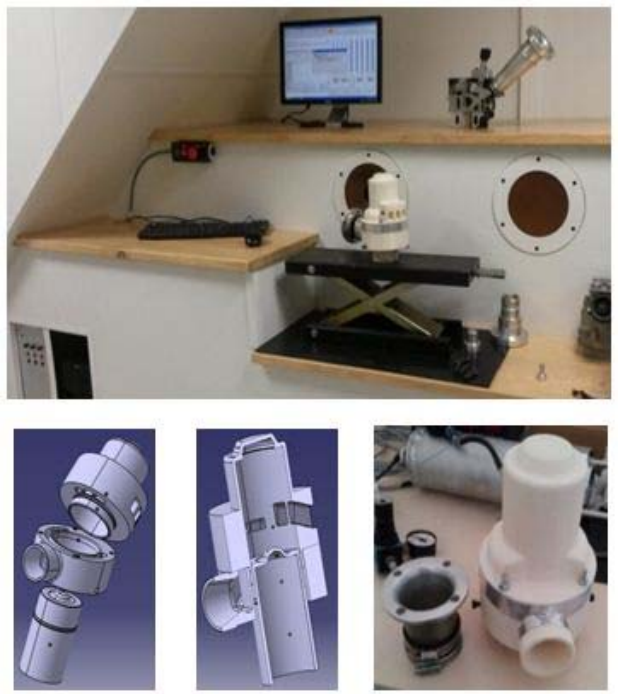

Fig. 3. Wind tunnel and experimental cylinder.

and temperatures. For the trials, the intake

overpressure varies from $10 \mathrm{mbar}$ to $30 \mathrm{mbar}$ with a 5 mbar step.

\subsection{Experimental results}

In the wind tunnel, the mass flow rate is calculated according to pressure and temperature measurements by the following equation:

$\dot{m}=S \sqrt{\frac{\mathrm{P}_{0} \times 100}{\mathrm{r}}} \sqrt{\frac{2 \times \mathrm{P}_{\text {nozzle }} \times 100}{\left(\mathrm{~T}_{\text {in }}+273\right)}}$

with $\mathrm{S}$ the nozzle sectional area $\left(\mathrm{m}^{2}\right), \mathrm{T}_{\text {in }}$ the temperature of the flow entering the cylinder $\left({ }^{\circ} \mathrm{C}\right)$, $\mathrm{P}_{0}$ the pressure of the fluid entering the nozzle (mbar) and $\mathrm{P}_{\text {nozzle }}$ the pressure of the fluid outgoing the nozzle (just before entering the cylinder). The specifcideal gas constant $\mathrm{r}$ is equal to 287 $\mathrm{J} \cdot \mathrm{kg}^{-1} \cdot \mathrm{K}^{-1}$ for air.

The results measured in the wind tunnel are given in Table1:

Table1 Experimental results of wind tunnel

\begin{tabular}{|c|c|c|}
\hline $\begin{array}{c}\text { Overpress } \\
\text { ure } \\
\text { (mbar) }\end{array}$ & $\begin{array}{c}\text { Mass flow } \\
\text { rateBDC }(\mathrm{g} / \mathrm{s})\end{array}$ & $\begin{array}{c}\text { Mass flow rate } \\
\text { middle point } \\
(\mathrm{g} / \mathrm{s})\end{array}$ \\
\hline 10 & 25.65 & 21.75 \\
\hline 15 & 31.57 & 26.61 \\
\hline 20 & 36.83 & 30.86 \\
\hline 25 & 41.46 & 34.71 \\
\hline 30 & 45.95 & 38.16 \\
\hline
\end{tabular}

Knowing the accuracy of sensors and Eq. (13), the errors of measurements are under $0.1 \%$. 


\subsection{Numerical results}

The iterative model ran until allresiduals (errors) dropped under $10^{-3}$.

The results given by the numerical model are expressed in Table2:

Table2Numerical results of wind tunnel

\begin{tabular}{|c|c|c|}
\hline $\begin{array}{c}\text { Overpress } \\
\text { ure (mbar) }\end{array}$ & $\begin{array}{c}\text { Mass flow rate } \\
\text { BDC }(\mathrm{g} / \mathrm{s})\end{array}$ & $\begin{array}{c}\text { Mass flow rate } \\
\text { middle point } \\
(\mathrm{g} / \mathrm{s})\end{array}$ \\
\hline 10 & 25.11 & 20.67 \\
\hline 15 & 31.61 & 25.71 \\
\hline 20 & 36.48 & 29.34 \\
\hline 25 & 41.22 & 32.96 \\
\hline 30 & 44.64 & 37.29 \\
\hline
\end{tabular}

\subsection{Comparison of results}

The validation of the numerical model thanks to experiments fits into the process of qualification. The method used by the authors is based on the PEPS method (Parsimony, Accuracy, Precision, Specialization) presented by Ordaz-Hernandez et al. (2007).

The comparison of numerical and experimental results is done in order to qualify the accuracy of the numerical model. The other criteria are not determined by experiment and some of them are evaluated in Caginet al. (2014). The results are plottedinFig. 4.

The more the flow is disturbed (when the piston covers a part of the ports), the more the difference between the results increases. At BDC, there is no major disturbing element (except for any change in the flow direction imposed by the geometry). The numerical model provides results almost identical to the experimental ones. At middle point, the piston masks a part of the ports which creates recirculation zones just in front of the piston and acceleration zones when the flow goes through the ports. Therefore, the kinetic energy in these zones is higher when the piston half-covers the ports than when it is at $\mathrm{BDC}$.

In average, the difference between experimental and numerical results is $1.3 \%$ at $\mathrm{BDC}$ and $4.1 \%$ at middle point. These differences are very low and they can be explained by the simplifying assumptions of the numerical model.

The aim of the study is to model the scavenging process as a whole in order to determine the overall characteristic quantities of the flow. Local phenomena are not the purpose, especially local turbulences. Thus, despite of the gap between the results and knowing the model assumptions, an error under $5 \%$ on the mass flow rate is an acceptable value which validates the numerical approach.

\section{PARAMETRICAL STUDY}

As previously explained, CFD simulations are not well adapted to determine the optimized solution ofaproblem because of its costs. 0D systemic modellings are very useful to simulate and to compare different configurations; but the $0 \mathrm{D}$ scavenging model is currently unrealistic such as perfect displacement and perfect mixing models (Hopkinson, 1914), 3-zones model (Maekawa, 1957) or "3-zones and multiphases" model (Benson and Whitehouse, 1983). The aim of the study is to improve the $0 \mathrm{D}$ scavenging model developing a parametric model based on CFD results.
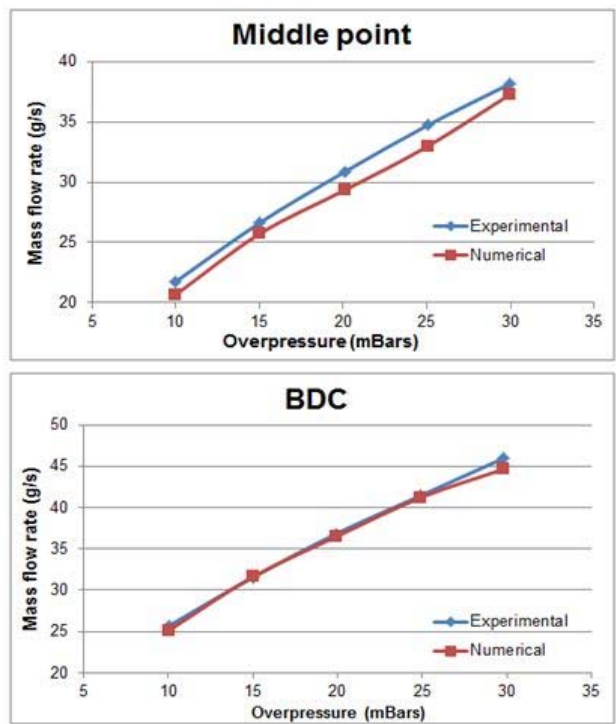

Fig. 4. Comparison of experimental and numerical results.

After defining the varying parameters and their possible values, several CFD models with different configurationshave run to observe the scavenging efficiency according to the value of parameters.

\subsection{Geometrical and environmental variables}

The influence of some design variables has been widely studied by others authors. The studies are, in majority, focused on only one parameter such as the orientation of the ports (Litke, 2000) or the short-cutting (Honget al., 2004). Taking into account the engine complexity, a multi-parametrical study has been carried out. The most influent parameters on the scavenging process and their range of values are presented by Caginet al. (2014). They are reminedbelow. The simplification of the geometry in 2D leads to focus on the influence of only nine variables:

- $\quad$ Angle $\beta$ (angle between the piston plane at BDC and the port),as shown in Fig. 5,

- Height of the exhaust port $\theta_{\text {end exh }}$ (in opposition of the intake ports, the exhaust port can be fully uncovered before the piston reaches the $\mathrm{BDC}$; it is expressed as the crankshaft angle when the exhaust port is fully opened),

- $\quad$ Advance of intake opening $\theta_{\text {in_adv }}$ (crankshaft 
angle at which the piston begins to uncover the port; all intake ports have the same open angle),

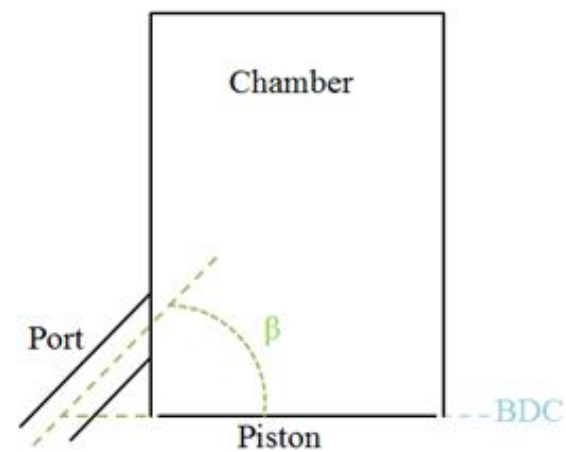

Fig. 5. Angle $\beta$.

- Advance of exhaust opening $\theta_{\text {exh_adv }}$ (crankshaft angle at which the piston begins to uncover the exhaust port),

- $\quad$ Boost pressure $P_{\text {boost }}$ (pressure of the intake gases),

- Difference between intake and exhaust pressures $\Delta \mathrm{P}$ (the exhaust back pressure is due to the turbine. Rather to talk about 'exhaust back pressure', the difference between the boost pressure and the exhaust back pressure is used),

- Intake temperature $\mathrm{T}_{\mathrm{in}}$,

- Engine velocity $\mathrm{V}_{\text {engine }}$.

The range of these different variables are summarized in Table 3.

Table 3 Design and environmental variables

\begin{tabular}{|c|c|}
\hline Symbol & Range of value \\
\hline$\theta_{\text {end_exh }}$ & $0-30 \mathrm{CAD}^{*}$ \\
\hline$\theta_{\text {exh_adv }}$ & $65-85 \mathrm{CAD}^{*}$ \\
\hline$\theta_{\text {in_adv }}$ & $35-55 \mathrm{CAD}^{*}$ \\
\hline $\mathrm{P}_{\text {boost }}$ & $1.5-4 \mathrm{bar}$ \\
\hline$\Delta \mathrm{P}$ & $0.2-0.6 \mathrm{bar}$ \\
\hline$\beta_{\text {in }}$ & $0-60$ degree \\
\hline$\beta_{\text {exhaust }}$ & $0-30$ degree \\
\hline $\mathrm{T}_{\text {in }}$ & $40-80^{\circ} \mathrm{C}$ \\
\hline $\mathrm{V}_{\text {engine }}$ & $2000-3000 \mathrm{rpm}$ \\
\hline
\end{tabular}

*before BDC.

\subsection{Results of the reference case}

The reference case is defined as follow:

- $\quad \beta_{\text {in }}=\beta_{\text {exh }}=0$ : no sloping duct which is the simplest geometry, the first geometry used,

- $\quad \theta_{\text {end_exh }}=0$ : intake and exhaust ducts are facing each other,

- $\quad \theta_{\text {in adv }}=35 \mathrm{CAD}$ and $\theta_{\text {exh adv }}=65 \mathrm{CAD}$ : the "power" phase is the longest as possible and the opening of intake port is far enough from the opening exhaust port to limit backflow,

- $\quad \mathrm{P}_{\text {boost }}=1.5$ bar and $\Delta \mathrm{P}=0.2 \mathrm{bar}$,

- $\quad \mathrm{T}_{\text {in }}=333.15 \mathrm{~K}$ and $\mathrm{V}_{\text {engine }}=2500 \mathrm{rpm}$.

A qualitative analysis of the numerical results is presented here below.

When the exhaust opens, the pressure in the cylinder is above 8bar which is suitable for the exhaust of the burnt gases through the port. After $30 \mathrm{CAD}$, the intake port opens. In the cylinder, the pressure is around $1.8 \mathrm{bar}$. The pressure inside the cylinder is higher than the intake pressure: some backflowsoccur which is clearly visible on the species distribution at $\theta=148 \mathrm{CAD}$ (cf. Fig. 6).
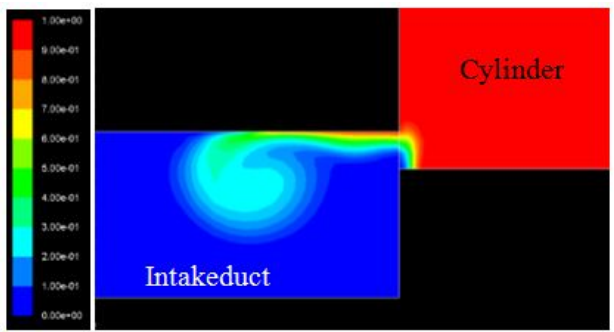

Fig. 6. Mass fraction of burnt gases just after intake port opening.

At $150 \mathrm{CAD}$, five degrees after intake opening, the pressure in intake ductis enough to boost the flow of fresh gases in the cylinder (cf. Fig. 7).
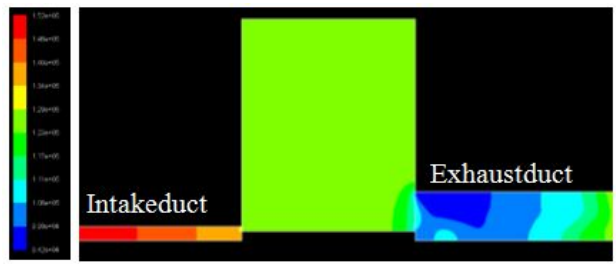

Fig. 7. Pressure field (in bar) at $\theta=150$ CAD.

At the end of the scavenging process, the average pressure in the chamber is $1.4 \mathrm{bar}$ (cf. Fig. 8). Because the intake port closes $30 \mathrm{CAD}$ before the exhaust one, the pressure in the cylinder is always under the boost pressure.

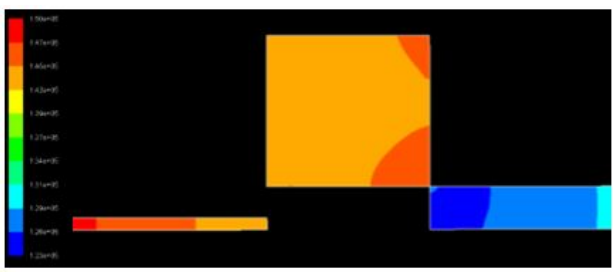

Fig. 8. Pressurefield(in bar) at $\theta=245$ CAD.

The opening advance of ports must be chosen very carefully. There are three main constraints to keep in mind. Firstly, if the exhaust port opens too early, the duration of compression and the work transmitted to the crankshaft will not be enough. Secondly, the intake must open early enough to 
enter sufficiently fresh gases for the next combustion. And thirdly, if the intake port opens too soon after exhaust opening, the cylinder pressure will remain too high and some backflow will occur. But, the larger the delay between the two closing (same as opening delay) is, the more importanttheshort-cutting is and the less high the pressure is inside the cylinder at the scavenging end. Knowing all these constraints, the choice of opening advance angles is a compromise to find.The flow structure is tightly linked to the aerodynamics in the chamber at the end of the combustion process.But the combustion process is not modeled so the simulation is initialized with no flow motion or turbulence in the cylinder at the beginning.

Fig 9displays the pathlines of the flow at $180 \mathrm{CAD}$ and severalphenomena are illustrated. The short-cutting is easily identifiable with the pathlines crossing the cylinder directly from the intake port to the exhaust one. The tumble motion is also illustrated in Fig. 9. Moreover, two recirculation areas appear after the flow separates in two: a part of gases goes through the exhaust port whereas the other part stays in the cylinder. They are the result of the separation of the flow from the wall.
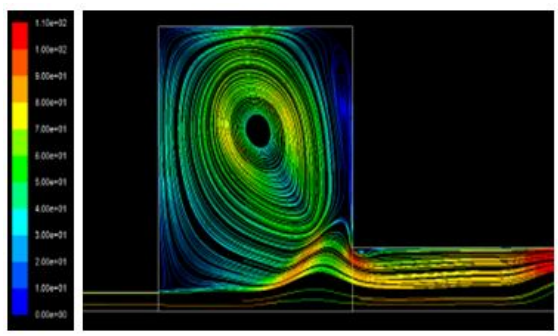

Fig. 9. Velocity pathlines(in $\mathrm{m} / \mathrm{s})$ at $\theta=180 \mathrm{CAD}$.

Large values of the turbulent kinetic energy appear when the exhaust port opens (cf.Fig. 10). The important difference of pressure creates very fast flow through the port and in the duct which is the cause of turbulent kinetic energy production.
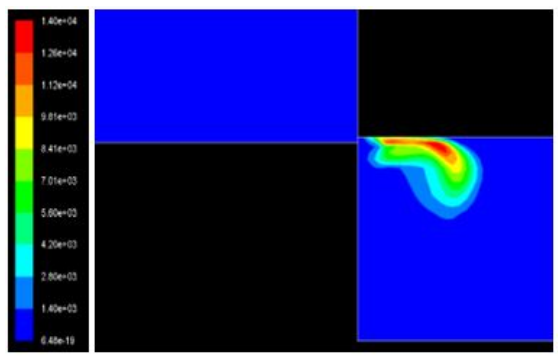

Fig. 10. Turbulent kinetic energy $k\left(\mathrm{~m}^{2} / \mathrm{s}^{2}\right)$ at $\theta=115.8$ CAD.

All throughout the simulation, the turbulent kinetic energy field is very closed to the velocity field and recirculated flow areas. Especially, the turbulent kinetic energy is high where the velocity is also important and where there are singular changes of flow direction (in the chamber, just before the exhaust duct), as seen inFig. 11 .

At the end of the scavenging process, a tumble motion of the flow is clearly present in the cylinder. The maximumflowvelocity in the cylinder is $60 \mathrm{~m} / \mathrm{s}$ and the averaged velocity is $30 \mathrm{~m} / \mathrm{s}$ : this is far under maximum velocity conditions during the scavenging. The averaged turbulent kinetic energy is low $\left(86 \mathrm{~m}^{2} / \mathrm{s}^{2}\right)$ thanks to the global regular motion of the flow in the chamber.
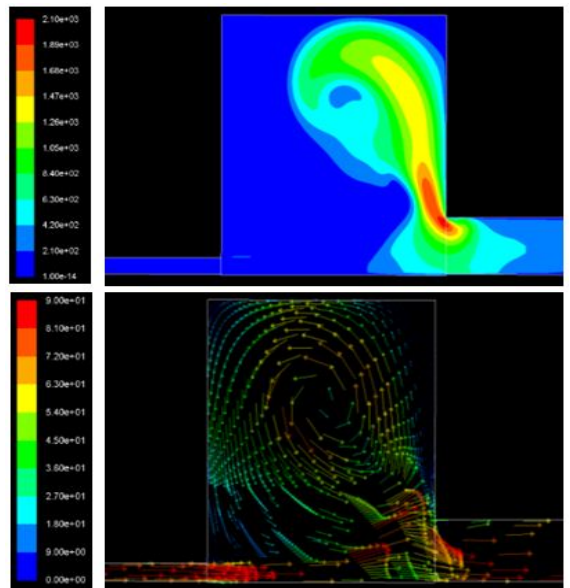

Fig. 11. Turbulent kinetic energy $k$ (in $\mathrm{m}^{2} / \mathrm{s}^{2}$ ) (up) and velocity field (in $\mathrm{m} / \mathrm{s}$ ) (down) at $\theta=180 \mathrm{CAD}$.

At the beginning of the simulation, there are only fresh gases in intake duct and only burnt gases in cylinder and exhaust duct.

Fig. 12shows the distribution of species during scavenging. At BDC, the fresh gases have started the loop in the cylinder. But the short-cutting is really important with this configuration: at $\theta=208$ $\mathrm{CAD}$, we observe that the most part of entering fresh gases goes directly to the exhaust and the mixing of species in cylinder is important. At the end of scavenging, the mass fraction of fresh gases is equal to 0.3 in the cylinder due to large short-cutting and species mixing. The short delay of intake port opening also explains the low proportion of fresh gases. The scavenging process is totally inefficient and we can assume that the mass of fresh gases will not be sufficient for the next step of combustion.

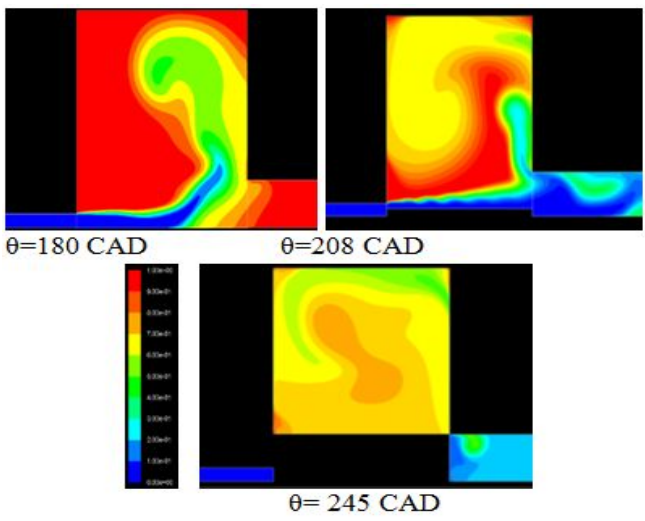

Fig. 12. Mass fraction of burnt gases. 
The basic design of the chamber and the scavenging results underline the need to improve cylinder design. Design improvements can be determined as soon as all design parameters involved in the process are defined and the objective variables are clearly evaluated.

\subsection{Objective variables}

In order to quantify and to qualify the scavenging process, the data recovery mainly relates to the mass of fresh and burnt gases. It is necessary to know:

- The mass of fresh gases entering the cylinder, $\mathrm{m}_{\mathrm{FG} \_ \text {in }}$,

- The mass of fresh gases which is short-cutted, $\mathrm{m}_{\mathrm{FG} \_ \text {exh }}$,

- The mass of burnt gases leaving the cylinder through intake ports (backflow), $\mathrm{m}_{\mathrm{BG} \_ \text {in }}$

- The mass of burnt gases leaving the cylinder through exhaust ports, $\mathrm{m}_{\mathrm{BG} \text { exh }}$.

The mass of burnt and fresh gases going through the port is known at each recorded time step. The data are exported every 22 time steps ( 1 time step lasted $3.10^{-6} \mathrm{~s}$ ) which is equivalent to $0.99 \mathrm{CAD}$. The time step has been carefully chosen after trials. Beyond $3.10^{-6} \mathrm{~s}$, the calculations are divergent (Caginet al., 2014): the piston displacement is too important to have a stable convergence of the equations towards a solution. A lower time step would increase the duration of the simulation which is not suitable.

The flow through the ports is considering constant between two data exports to reduce the data quantity and time needed to analyze it. A python program evaluates total masses entering and leaving the cylinder according to the time(in second) between two data recovery and the openness of the ports (linked to the piston position also calculated by the program).

Thanks to the python program, the masses of burnt and fresh gases going through each port are known. The objective variables can be now easily determined.

To compare the scavenging efficiency in different cylinder designs, four main parameters introduced by Heywood (1988) are used:

- The delivery ratio:

$\Lambda=\frac{\text { mass of delivered air per cycle }}{\text { reference mass }}$

- The trapping efficiency:

$\eta_{t r}=\frac{\text { mass of delivered air retained }}{\text { mass of delivered air }}$

- The scavenging efficiency:

$\eta_{s c}=\frac{\text { mass of deliverd air retained }}{\text { mass of trapped cylinder charge }}$

- The charging efficiency: $\eta_{c h}=\frac{\text { mass of deliverd air retained }}{\text { displaced volume } \times \text { ambient density }}$

The trapping and charging efficiencies are linked to the delivery ratio by the following equation:

$\eta_{s c}=\Lambda \times \eta_{t r}$

The delivery ratio quantifies the difference between the theoretical and the real entering mass of fresh gases. The trapping efficiency evaluates the quantity of fresh gases which enters and is still in the cylinder after the port closure. The scavenging efficiency underlines the quantity of burnt gases replaced by fresh ones. Finally, the charging efficiency quantifies the difference between the theoretical entering mass of fresh gases and the trapping mass of fresh gases in the cylinder.

These parameters are widely used in motoring to compare different designs and to determine the optimized one which is done in the next section.

\subsection{Factorial design and simulations}

To study the influence of parameters defined in section 3.1, the value of each parameter varies following a statistical method called fractional factorial designs. This approach extracts a maximum of information from a minimum of experiments. The level of each variable is fixed to 3 (the two extremes of each range of value plus the median value).

Considering the important number of variables involves in the scavenging process, we had to set the values of two variables: the engine velocity (set to 2500rpm) and the intake gases temperature (set to $333 \mathrm{~K})$.

A statistical treatment of the results (analysis of effects and analysis of variance) has been done. This treatment is useful to determine which parameter is the most influential over each objective variable. To reach the optimal value of each objective variable, the designer has to find the suitable value of the parameter which has the most of influence. In our case of study, the control of the two most influential parameters represents the control of more than $65 \%$ of each objective variable. All the analysis results are presented in Table 4 . The most influential parameter over each objective variable is in green.

Table 4 Influence of parameters over objective variable

\begin{tabular}{|c|c|c|c|c|}
\hline & $\Lambda$ & $\eta_{\text {tr }}$ & $\eta_{\text {ch }}$ & $\eta_{\text {sc }}$ \\
\hline $\begin{array}{c}\theta_{\text {end_e }} \\
\text { xh }\end{array}$ & $1.88 \%$ & $9.90 \%$ & $13.38 \%$ & $11.80 \%$ \\
\hline $\begin{array}{c}\theta_{\text {exh_a }} \\
\text { dv }\end{array}$ & $2.30 \%$ & $2.29 \%$ & $3.74 \%$ & $3.31 \%$ \\
\hline$\theta_{\text {in_adv }}$ & $56.43 \%$ & $0.38 \%$ & $58.47 \%$ & $63.14 \%$ \\
\hline$P_{\text {boost }}$ & $12.39 \%$ & $0.96 \%$ & $3.50 \%$ & $1.63 \%$ \\
\hline$\Delta \mathrm{P}$ & $10.56 \%$ & $8.85 \%$ & $1.97 \%$ & $0.23 \%$ \\
\hline$\beta_{\text {in }}$ & $13.85 \%$ & $75.03 \%$ & $13.06 \%$ & $15.76 \%$ \\
\hline$\beta_{\text {exh }}$ & $2.59 \%$ & $2.56 \%$ & $5.89 \%$ & $4.13 \%$ \\
\hline
\end{tabular}


It is very interesting to note that only two parameters are significantly influential: the advance of intake opening (significant parameter for 3 of the fourth objective variables) and the angle $\beta$ of the intake duct. Their influence is over $50 \%$ and all other parameters are under $16 \%$. This result confirms Taguchi hypothesis: only few parameters (one or two) have a real influence, all others are negligible. From an engine designer's point of view, the two parameters are totally unconnected. The trapping efficiency can be optimized separately of the delivery ratio and the scavenging and charging efficiencies.

In order to characterize the influence of the two parameters, two configurations are compared. The two configurations were chosen because of their efficiencies (the worst and the best ones).

In the first configuration, $\theta_{\text {in_adv }}$ and $\beta_{\text {in }}$ are respectively equal to $55 \mathrm{CAD}$ (before $\mathrm{BDC}$ ) and 60CAD which are the maximum possible values. It has provided the best results: $\Lambda=1.21, \eta_{\mathrm{tr}}=0.79$, $\eta_{\mathrm{ch}}=0.97, \eta_{\mathrm{sc}}=0.66$.

In the second configuration, $\theta_{\text {in_adv }}$ and $\beta_{\text {in }}$ are respectively equal to $35 \mathrm{CAD}$ (before $\mathrm{BDC}$ ) and OCAD which are the minimum possible values. It has provided the best results: $\Lambda=0.22, \eta_{\mathrm{tr}}=0.03$, $\eta_{\mathrm{ch}}=0.01, \eta_{\mathrm{sc}}=0.03$.

The values of $\theta_{\text {in adv }}$ and $\beta_{\text {in }}$ for the best and the worst configurations seem to confirm that these two variables are very influent on the scavenging.

The velocity pathlinesin Fig. 13 shows that, in the case of the best configuration, the entering flow is guided towards the cylinder head. The path limits the short-cutting; and it favors the trapping of fresh gases and the exhaust of the burnt ones. On the other hand, with the worst configuration, the flow of fresh gases directly goes through the exhaust port which restraints the replacement of burnt gases by fresh ones. The distribution of species (cf.Fig. 14) also leads to the same conclusion.
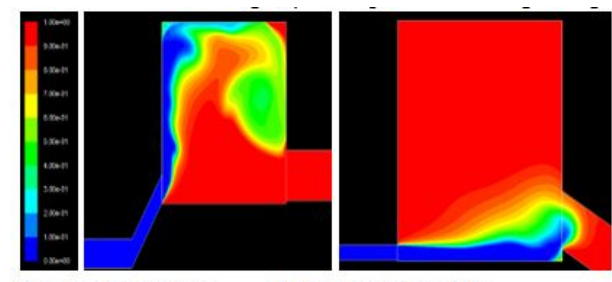

Best configuration

Worst configuration

Fig. 13. Velocity pathlines at $\theta=180 \mathrm{CAD}$.

Fig 14 underlines that the angle $\beta$ directly controls the scavenging flow direction. With the maximum value of $\beta$, the entering flow is guided towards the top of cylinder. Short-cutting is totally avoided before the piston reaches BDC and the bulk of the chamber is scavenged. On the contrary, with the worst configuration, the lack of sloping angle directly guides the fresh gases towards the exhaust port.

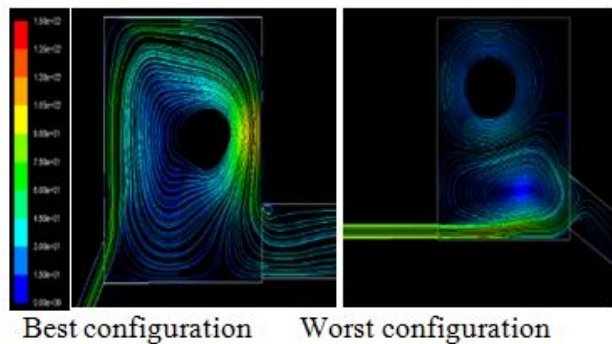

Fig. 14. Mass fraction of burnt gases at $\theta=180$

CAD.

The influence of angle $\beta$ was predictable regarding design evolutions of 2 -stroke engine (with ports). Intake and exhaust by ports were very common for moped engines. These engines especially offered low engine and scavenging efficiencies. To increase the performances, one of the first evolutions was to add deflectors on the piston head which has the same impact as sloping ducts.

The advance of intake opening directly impacts the intake port opening time: the longer this time is, the more important the entering mass of fresh gases is. This is confirmed by the simulations results: with the best configuration, 14.1g of fresh gases went through the intake port against only $6.7 \mathrm{~g}$ in the worst configuration (same boost pressure). The difference of fresh gases masses is accentuated by the significant short-cutting in the worst case.

\section{CONCLUSION}

This study presents first steps of a method to establish a tool to optimize the cylinder design integrating a realistic model of the scavenging process. But in order to determine the analytical scavenging model (integrating the influence of design parameters), a CFD study is essential as the creation of a data base. The data base contains the results of multiples CFD simulations with different values of parameters. This way, the scavenging model coming from the data base takes into account the influence of parameters. After integrating the scavenging model into a OD engine system, different geometries can be tested and design optimization can be done.

The study in $2 \mathrm{D}$ of the scavenging process and the parameters involved clearly highlights the importance of only two parameters: the angle $\beta$ of the intake duct and the opening angle of the intake port. They determine more than $65 \%$ of our objective variables.During the design process, the values of these two variables must be first determined. The values of the others variable are less important but they still need to be carefully fixed in order to optimize the global design.

\section{REFERENCES}

Benson, R. S. and N. D. Whitehouse (1983). Internal Combustion Engines. Pergamon Press. 
Cagin, S., X. Fischer, N. Bourabaa, E. Delacourt, C. Morin, and D. Coutellier (2014, August). A Methodology for a New Qualified Numerical Model of a 2-Stroke Diesel Engine Design. In Proceedings of International Conference On Advances in Civil, Structural and Mechanical Engineering - CSME, Hong-Kong, China.

Ghoje, J. (1997, December). Analytical Heat Release Function for DI Diesel Engines. In Proceedings of the Fourth Asian-Pacific International Symposium on Combustion and Energy Utilization, Bangkok, Thailand.

Heywood, J. B. (1988).Internal Combustion Engines Fundamentals. McGraw-Hill Education.

Hopkinson, B. (1914). The Charging of Two-Cycle Internal Combustion Engines. Journal of the American Society for Naval Engineers 3, 974-985.

Hong, G., A. N. F. Mack, F. Menolotto, C. S. Jamieson and S.G. Main (2004). Numerical Visualization of Air Short-Circuiting in a Small Two-Stroke SI Engine. SAE Technical Paper 2004-32-0009.

Ingvorsen, K. M., K. E. Meyer, T. Schnipper, J. H. Walther, and S. Mayer (2012, July). Swirling Flow In Model Of Large Two Stroke Diesel Engine. In Proceedings of $16^{\text {th }}$ International Symposium on Applications of Laser Techniques to Fluid Mechanics, Lisbon, Portugal.

Kato, S., H. Nakagawa, K. Kawahara, T. Adachi, and M. Nakashima (1991). Numerical Analysis of the Scavenging Flow in a Two-Stroke-Cycle Gasoline Engine. JSME International Journal, Fluids Engineering, Heat Transfer, Power, Combustion, Thermophysical Properties 34, 385-390.

Lamas Galdo, M. I. and C. G. Rodríguez Vidal (2012a). Computational Fluid Dynamics Analysis of the Scavenging Process in the MAN B\&W 7S50MC Two-Stroke Marine Diesel Engine. Journal of Ship Research 56, 154-161.

Lamas Galdo, M. I. and C.G. Rodríguez Vidal (2012b). Simulation of the Scavenging Process in Two-Stroke Engines.Numerical Modelling.InTech.

Litke, B. (2000). The Influence of Inlet Angles in Inlet Ports on the Scavenging Process in Two-Stroke Uniflow-Scavenged Engine.
Mrine TechnologyIII, Marine and Maritime 1, 247-252.

Maekawa, M. (1957), Text of Course. JSME G36, 23.

Mattarelli, E. (2009). Virtual Design of a Novel Two-Stroke High-Speed Direct-Injection Diesel Engine. International Journal of Engine Research 10, 175-193.

Noor, M.M., K. Kadirgama and R. Devarajan (2008, December). CFD Simulation and Validation of the In-Cylinder within a Motored Two Stroke SI Engine. In Proceedings of $2^{\text {nd }}$ International Conference on Science and Technology, Penang, Malaysia.

Ordaz-Hernandez, K., X. Fischer and F. Bennis (2007). A Mathematical Representation for Mechanical Model Assessment: Numerical Model Qualification Method. International Journal of Mathematics Sciences 1, 216-226.

Pitta, S.R., and R. Kuderu (2008). A Computational Fluid Dynamics Analysis on Stratified Scavenging System of Medium Capacity Two-Stroke Internal Combustion Engines. Thermal Science 12, 33-42.

Prasad, B.V., C. S. Sharma, T. N. C Anand and R.V. Ravikrishna (2011). High Swirl-Inducing Piston Bowls in Small Diesel Engines for Emission Reduction. Applied Energy 88, 2355-2367.

Shih, T.H., W. W. Liou, A. Shabbir, Z. Yang and J. Zhu (1994). ANew k- $\varepsilon$ Eddy Viscosity Model for High Reynolds Number Turbulent Flows: Model Development and Validation.NASA Technical Memorandum 106721.

Sigurdsson, E. (2011). Scavenging Flow in a Two-Stroke Diesel Engine. Ph. D. thesis, Technical University of Denmark, Denmark.

Swoboda, B. (1984). Mécanique Des Moteurs Alternatifs. Edition Technip.

Trescher, D. (2008). Development of an Efficient 3-D CFD Software to Simulate and Visualize the Scavenging of a Two-Stroke Engine. Archive of Computational Methods in Engineering 15, 67-111.

Zancanaro, F. V. and H .A. Vielmo (2010, July). Numerical Analysis of the Fluid Flow in a High Swirled Diesel Engine. In Proceedings of $7^{\text {th }}$ International Conference on Heat Transfer, Fluid Mechanics and Thermodynamics, Antalya, Turkey. 